\title{
Research Subjects and Research Trends in Medical Informatics
}

\author{
Kemal Hakan Gülkesen ${ }^{1}$ Reinhold Haux ${ }^{1}$ \\ 1 Peter L. Reichertz Institute for Medical Informatics, TU Braunschweig \\ and Hannover Medical School, Braunschweig, Germany \\ Methods Inf Med 2019;58:e1-e13. \\ Address for correspondence Kemal Hakan Gülkesen, MD, PhD, \\ Peter L. Reichertz Institute for Medical Informatics, TU Braunschweig \\ and Hannover Medical School, Mühlenpfordtstraße 23, 38106 \\ Braunschweig, Germany (e-mail: hgulkesen@gmail.com).
}

\begin{abstract}
Objectives To identify major research subjects and trends in medical informatics research based on the current set of core medical informatics journals.

Methods Analyzing journals in the Web of Science (WoS) medical informatics category together with related categories from the years 2013 to 2017 by using a smart local moving algorithm as a clustering method for identifying the core set of journals. Text mining analysis with binary counting of abstracts from these journals published in the years 2006 to 2017 for identifying major research subjects. Building clusters based on these terms for the complete time period as well as for the periods 2006-2008, 2009-2011, 2012-2014, and 2015-2017 for identifying trends.

Results The identified cluster includes 17 core medical informatics journals. By text mining of these journals, 224,992 different terms in 14,414 articles were identified covering 550 specific key terms. Based on these key terms five clusters were identified: "Biomedical Data Analysis," "Clinical Informatics," "EHR and Knowledge Representation," "Mobile Health," and "Organizational Aspects of Health Information Systems." No shifts in the clusters were observed between the first two 3-year periods. In the third period, some terms like "mobile phone," "mobile apps," and "message" appear. Also, in the third period, a "Clinical Informatics" cluster appears and persists in the fourth

Keywords

- medical informatics

- biomedical and health informatics

- research period. In the fourth period, a rearrangement of clusters was observed.

Conclusions Beside classical subjects of medical informatics on organizing, representing, and analyzing data, we observed new developments in the context of mobile health and clinical informatics. These subjects tended to grow over the past years, and we can expect this trend to continue.
\end{abstract}

\section{Background}

Medical informatics (MI), or more generally Biomedical and Health Informatics, has been most variously and often inconsistently defined. ${ }^{1}$ According to one definition, it is "concerned with the optimal use of information, often aided by the use of technology, to improve individual health, health care, public health, and biomedical research."2 According to another it is "a discipline, concerned with the systematic organization, representation, and analysis of data, information, and knowledge in biomedicine and health care." ${ }^{3}$ Recommendations for MI education, which were revised by the International Medical Informatics Association (IMIA), can also be a clue for defining the field of MI. ${ }^{4}$

On the other hand, MI is frequently referred to by other names, with different yet closely related meanings. "Biomedical and Health Informatics," "Biomedical Informatics," Healthcare Informatics," and "Clinical Informatics" are some of them. ${ }^{5}$ As the name MI is more frequently used in journal classifications (such as Institute for Scientific Information [ISI] and Science-Metrix), terminologies (PubMed), or in the names of non-governmental organizations (such as IMIA and American received

August 21, 2018

accepted after revision

January 7, 2019

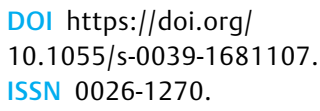

DOI https://doi.org/

10.1055/s-0039-1681107.

ISSN 0026-1270.

(c) 2019 Georg Thieme Verlag KG Stuttgart · New York
License terms

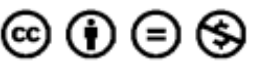


Medical Informatics Association [AMIA]), in the present text, we preferred to use MI as a term.

A systematic approach to help define MI could improve our understanding of its research contents by analyzing its patterns of communication through publications produced by the MI community. This would also help in designing and reshaping MI education. And it will additionally help to support management decisions and to design future research agendas. ${ }^{6}$

Several studies have been published examining the MI literature. To select MI articles or journals, the authors of these studies mainly used four approaches.

- Using the Medical Subject Headings (MeSH) indexing to define MI articles. ${ }^{7-11}$

- Composing a core MI journal set by expert opinions. ${ }^{12}$

- Clustering journals by co-citation data to determine a core MI journal set. ${ }^{13}$

- Text mining of abstracts and clustering journals by the help of the obtained terms to determine a core MI journal set. ${ }^{14}$

We also wanted to examine research subjects in MI. Our approach is to use footprints of scientific knowledge: references. Direct citation (intercitation), ${ }^{15}$ bibliographic coupling, ${ }^{16}$ and co-citation ${ }^{17,18}$ are three main approaches for clustering similar articles or journals. When an article cites another article, the relationship is denoted as direct citation. When two different articles cite an article, the relationship is called bibliographic coupling. When articles from two different journals are included in the same reference list, it is called co-citation ( - Fig. 1). ${ }^{13,19}$ Direct citation was reported to be more successful than the other two methods for clustering similar articles in the analysis of historical data. ${ }^{20}$ However, bibliographic coupling is possibly better in relatively short-term data analysis. ${ }^{15,20}$

A scientific community communicates its research in its scientific journals, and the contents of these journals reflect the main areas of interest of the community. The content of the MI literature has been examined in several earlier papers,

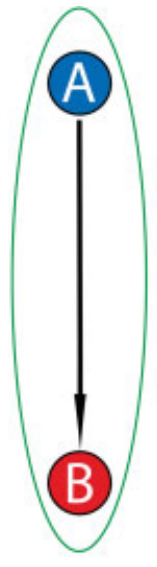

Direct citation

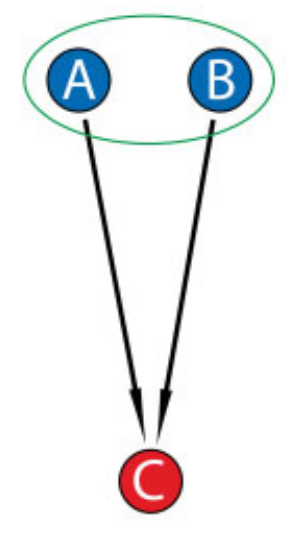

Bibliographic coupling

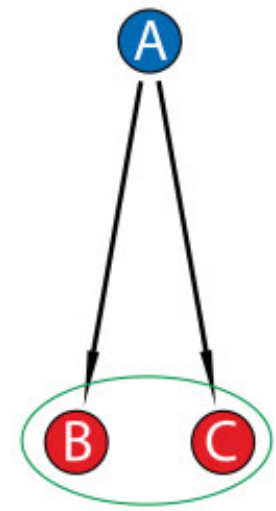

Co-citation
Fig. 1 Visual representation of citation relations. A, B, and C represent different journals and arrows represent citations. Green ovals show how the documents are clustered by each approach. with authors taking two main approaches to classify or cluster the content of MI articles or journals.

- Documentation of MeSH terms used for indexing the articles. $9,11,12,21$

- Extraction of terms by text mining of the abstracts. ${ }^{10,14}$

Another approach involves examining an author's choice of keywords. ${ }^{22}$ However, both MeSH terms and author keywords are affected by human factors involving subjective bias. In the present study, we preferred text mining as a somewhat more objective method for examining and finding possible groupings for the MI literature.

Some of the previous studies divided the literature into time periods to make comparisons between them, and to identify trends. ${ }^{9,11,12,14}$ We also examine publications across different time periods to examine and compare their contents.

\section{Objectives}

Two questions motivated us to conduct this study:

$\mathrm{Q}_{1}$ : What are the major research subjects in MI?

$\mathrm{Q}_{2}$ : Do these subjects change over time? If they change, what do these changes look like?

Before being able to provide answers to these questions, another question arose. Assuming that MI research is often communicated through core MI journals:

$\mathrm{Q}_{0}$ : What might be the current core MI journals?

The third and fourth sections concentrate on $Q_{0}$, while our main questions, $Q_{1}$ and $Q_{2}$, will be examined in the fifth and sixth sections.

\section{Study Design, Methods, and Tools for Identifying Core Medical Informatics Journals $\left(\mathbf{Q}_{\mathbf{0}}\right)$}

For the years 2013 to 2017, we considered all journals listed in the Web of Science (WoS) under the categories "Medical Informatics," "Biochemical Research Methods," "Biotechnology and Applied Microbiology," "Mathematical and Computational Biology," "Statistics and Probability," "Computer Science: Information Systems," "Health Care Sciences and Services," "Engineering: Biomedical," "Computer Science: Interdisciplinary Applications," "Computer Science: Theory and Methods," "Computer Science: Artificial Intelligence," and "Public, Environmental and Occupational Health." We included all papers of all journals that published 40 or more articles during this time period. The data were downloaded between June 4 and June 18, 2018. The tool used for clustering was VOSviewer (version 1.6.8). ${ }^{23}$ The reference data were extracted automatically by VOSviewer. As clustering technique the smart local moving algorithm, introduced by Waltman and Van Eck, ${ }^{24}$ was used with bibliographic coupling analysis and fractional counting. ${ }^{25}$ VOSviewer also enabled visualizing the obtained results. For this visualization, association strength was used as the normalization method, because it was recommended for bibliometric studies. ${ }^{26}$ Because too large clusters were obtained by using the default resolution value (1.00), it was increased to 4.50 . We tested 
the most satisfactory clustering by 0.5 increments in resolution value. At level 4.0 there were 39 clusters and most of the MI journals, such as JAMIA, were in the largest cluster, containing 89 journals, mostly health management journals. At level 5.0 there were 54 clusters. The MI cluster was composed of 16 journals, with all journals being the same as level 4.5, except Artificial Intelligence in Medicine, which was in another cluster as a single journal. We considered 4.5 as the most satisfactory resolution value. Attraction and repulsion values (these values do not affect results but aesthetic appearance of figures) were taken as 2 and 0 , respectively. The authors selected and named related clusters independently and then came together to reach a consensus decision on the final naming of these clusters. The first author (K.H.G.) received his PhD 9 years ago and has 14 years of MI research and teaching experience in MI departments of universities. The second author (R.H.) received his PhD 35 years ago. He has been working as a university professor in MI departments of universities for 31 years, and wrote several MI textbooks. Both of the authors are or have been in editorial boards of various journals. Most of these journals are in the field of MI.

This research does not involve human subjects, human material, or human data.

\section{Results for Identifying Core Medical Informatics Journals $\left(\mathbf{Q}_{\mathbf{0}}\right)$}

Downloaded data amounted to a total of 427,012 articles, published in 867 journals, of which 807 contained 40 or more articles.

Forty-seven clusters were obtained (see Online Supplementary Material 1), and one of them included the core MI journals (-Fig. 2). This MI cluster includes 15 journals out of the 25 journals in the MI category in WoS, plus two additional telemedicine journals ( - Table 1 ).

As related clusters, we defined those clusters being close to the identified MI cluster, the clusters containing journals of the WoS MI category, and the cluster containing bioinformatics journals. Six related clusters have been found and named: "Bioinformatics," "Biomedical Engineering: Imaging and Information Technology," "Biomedical Engineering: Biomechanics and Medical Technology," "Health Management," "Information Management," and "Statistics" (-Fig. 2). For these six journal clusters, the authors independently gave the same names to "Bioinformatics," "Statistics," "Health Management," and "Information Management" clusters (66.7\% initial agreement rate) and two clusters of biomedical engineering were named by a consensus decision.

\section{Study Design, Methods, and Tools for Identifying Subjects and Trends}

For the 17 core MI journals identified we downloaded the abstracts of all these journals from articles published from the years 2006 to 2017 in the WoS-on July 5, 2018. We searched only "article" type documents in "Science Citation Index Expanded." We performed text mining analysis with binary counting to obtain terms. Terms were one to four word expressions. Then we were clustering these terms by using the smart local moving algorithm with the co-word method with binary counting. ${ }^{27}$

As the tool for text mining and co-word clustering we used VOSviewer (version 1.6.8), ${ }^{18}$ which was also used for visualizing the results. Weights of links, weight of total link strength, and weights of occurrences for each term are given in online supplementary materials. For explanation of meanings of these weights, please refer VOSviewer Manual. ${ }^{28}$

The text mining module of VOSviewer is based on the Apache OpenNLP toolkit. ${ }^{29}$ Its text mining functionality is described in the work of Van Eck and Waltman. ${ }^{30}$ Text mining functionality of VOSviewer does not need preprocessing. It automatically imports abstracts and processes data in five steps: (1) removal of copyright statements; (2) sentence detection; (3) part-of-speech tagging (using this algorithm, each word is assigned a part of speech, such as verb, noun, adjective, preposition, and so on); (4) noun phrase identification (it defines a noun phrase as a sequence of one or more consecutive words within a sentence such that the last word in the sequence is a noun and each of the other words is either a noun or an adjective); and (5) noun phrase unification (unification of noun phrases is accomplished by removing most nonalphanumeric characters, by removing accents from characters, by converting upper case characters to lower case, and by converting plural noun phrases to singular). For visualization of our results, association strength was used as a normalization method. The resolution parameter value was chosen as 1.00 , and the attraction and repulsion parameter values were taken as 2 and 1 , respectively.

We selected frequently used terms-those which were found in at least 50 articles (and also used 25 as noted below). The authors gave names to the clusters independently and then came together to reach a consensus on the final naming of these clusters.

We divided the articles into four groups, each covering a 3-year interval: 2006-2008, 2009-2011, 2012-2014, and 2015-2017. We analyzed each group in the same way as mentioned above. We selected those terms which are used in at least 25 articles. Resolution values for clustering were set as $1.00,1.10,1.20$, and 1.20 for the periods, respectively. Result of the default resolution value 1.0 was satisfactory for us in the first period, but we needed to increase the resolution values in the following periods to obtain similar clusters. This may be due to increasing number of articles with time.

This research does not involve human subjects, human material, or human data.

\section{Results for Identifying Subjects and Trends}

\section{On Major Research Subjects in medical informatics}

$\left(Q_{1}\right)$

By text mining of abstracts of articles from the 17 core MI journals, we detected 224,992 different terms in 14,414 articles. We scanned all terms, which were used in more than 50 articles and found the terms with the same or similar 

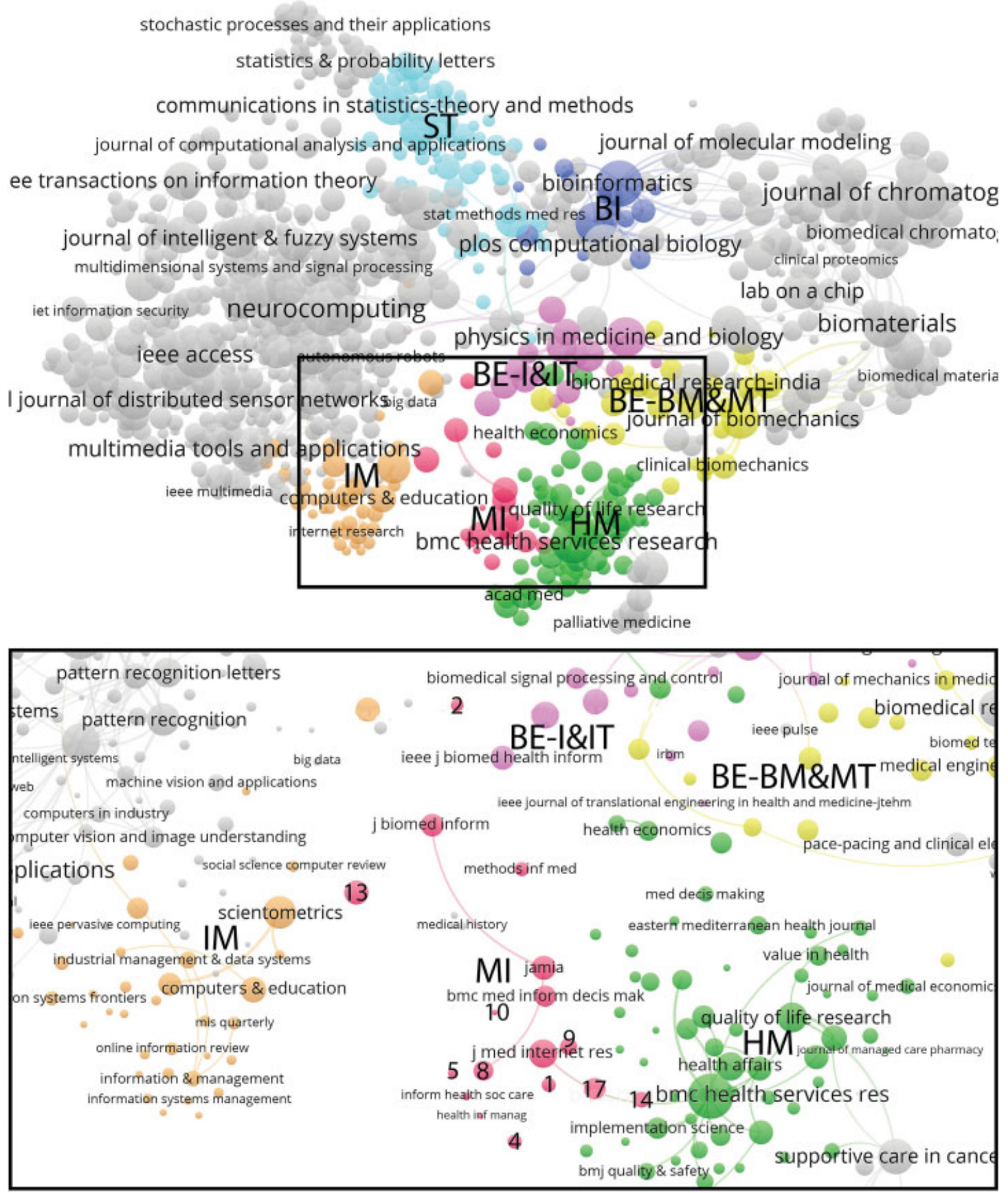

Fig. 2 Visualization of the journal clusters according to our analysis. Red: MI, green: HM, Health Management, orange: IM, Information Management, purple: BE-I\&IT, Biomedical Engineering: Imaging And InformationTechnology, yellow: BE-BM\&MT, Biomedical Engineering: Biomechanics And Medical Technology, blue: BI, Bioinformatics, and light blue: ST, Statistics. The other clusters are not colored. Journal names in the MI cluster (denoted by numbers): 1: Applied Clinical Informatics, 2: Artificial Intelligence in Medicine, 4: CIN-Computers Informatics Nursing, 5: Health Informatics Journal, 8: International Journal of Medical Informatics, 9: JMIR mHealth and uHealth, 10: JMIR Serious Games, 13: Journal of Medical Systems, 14: Journal of Telemedicine and Telecare, and 17: Telemedicine and e-Health. On the journal numbering, see also $=$ Table 1.

meaning. We converted the terms with the same or similar meaning to the most frequent one by the "replace by" function of VOSviewer to combine them as a single term (Online Supplementary Material 2). We obtained 1,334 such combined terms at the end of this process. A relevance score ${ }^{27}$ was calculated for each term, and 800 terms-that is, approximately $60 \%$ of the most relevant terms-were selected for manual processing. There we eliminated nonspecific terms (Online Supplementary Material 3). In the end, 550 distinct terms were obtained. Cluster analysis of these terms revealed five different clusters (Online Supplementary Material 4). We named these five clusters as (1) "Mobile Health," (2) "Organizational Aspects of Health Information Systems," (3) "Biomedical Data Analysis" (4) "EHR and 
Table 1 List of all journals in the identified cluster of core MI journals as well as of those journals belonging to the WoS MI category with their assignment to identified clusters

\begin{tabular}{|c|c|c|c|}
\hline Resulting clusters & & Journals & WoS categories \\
\hline \multirow[t]{17}{*}{$\mathrm{Ml}$} & 1 & Applied Clinical Informatics & $\mathrm{MI}$ \\
\hline & 2 & Artificial Intelligence in Medicine & $\mathrm{MI}$ \\
\hline & 3 & BMC Medical Informatics and Decision Making & $\mathrm{MI}$ \\
\hline & 4 & CIN-Computers Informatics Nursing & $\mathrm{Ml}$ \\
\hline & 5 & Health Informatics Journal & $\mathrm{MI}$ \\
\hline & 6 & Health Information Management Journal & MI \\
\hline & 7 & Informatics for Health and Social Care & $\mathrm{MI}$ \\
\hline & 8 & International Journal of Medical Informatics & $\mathrm{MI}$ \\
\hline & 9 & JMIR mHealth and uHealth & $\mathrm{Ml}$ \\
\hline & 10 & JMIR Serious Games & $\mathrm{MI}$ \\
\hline & 11 & Journal of Biomedical Informatics & $\mathrm{MI}$ \\
\hline & 12 & Journal of Medical Internet Research & $\mathrm{Ml}$ \\
\hline & 13 & Journal of Medical Systems & $\mathrm{MI}$ \\
\hline & 14 & Journal of Telemedicine and Telecare & HS\&S \\
\hline & 15 & Journal of the American Medical Informatics Association & $\mathrm{MI}$ \\
\hline & 16 & Methods of Information in Medicine & $\mathrm{MI}$ \\
\hline & 17 & Telemedicine and e-Health & HS\&S \\
\hline \multirow{4}{*}{\multicolumn{2}{|c|}{ Health management }} & International Journal of Technology Assessment in Health Care & $\mathrm{Ml}$ \\
\hline & & Journal of Evaluation in Clinical Practice & $\mathrm{MI}$ \\
\hline & & Medical Decision Making & $\mathrm{MI}$ \\
\hline & & Therapeutic Innovation and Regulatory Science & $\mathrm{Ml}$ \\
\hline \multirow{2}{*}{\multicolumn{2}{|c|}{$\begin{array}{l}\text { BE: Biomechanics and medi- } \\
\text { cal technology }\end{array}$}} & Biomedical Engineering/Biomedizinische Technik & $\mathrm{MI}$ \\
\hline & & Medical and Biological Engineering and Computing & $\mathrm{MI}$ \\
\hline \multirow{2}{*}{\multicolumn{2}{|c|}{$\begin{array}{l}\text { BE: Imaging and information } \\
\text { technology }\end{array}$}} & Computer Methods and Programs in Biomedicine & $\mathrm{Ml}$ \\
\hline & & IEEE Journal of Biomedical and Health Informatics & $\mathrm{Ml}$ \\
\hline \multirow{2}{*}{\multicolumn{2}{|c|}{ Statistics }} & Statistical Methods in Medical Research & $\mathrm{MI}$ \\
\hline & & Statistics in Medicine & $\mathrm{MI}$ \\
\hline
\end{tabular}

Abbreviations: BE, biomedical engineering; HS\&S, Healthcare Sciences \& Services; MI, medical informatics; WoS, Web of Science.

Knowledge Representation," and (5) "Clinical Informatics" (-Fig. 3). The authors gave the same names to all clusters except "EHR and Knowledge Representation" (80.0\% initial agreement rate).

The most frequent 24 terms in each cluster are presented in - Table 2.

\section{How Subjects can Change over Time $\left(Q_{2}\right)$}

The 2006 to 2008 Period

There were 402 terms in 2,001 articles and 241 of them were in the most relevant $60 \%$. After elimination of nonspecific terms, 142 of them were selected for cluster analysis. At the end of our analysis, we obtained four groups (Online Supplementary Material 5; - Fig. 4).

\section{Pattern of Clusters for the 2009 to 2011 Period}

In 2,765 articles in this period, there were 553 terms and 332 of them were in the most relevant $60 \%$. After nonspecific terms were eliminated, the remaining 205 terms were used for cluster analysis (Online Supplementary Material 6; - Fig. 5).

\section{Pattern of Clusters for the 2012 to 2014 Period}

There were 4,378 articles and 916 terms. A total of 550 were in the most relevant $60 \%$. After nonspecific terms were eliminated, the remaining 368 terms were used for cluster analysis. (Online Supplementary Material 7; -Fig.6).

\section{Pattern of Clusters for the 2015 to 2017 Period}

For 5,270 articles in this period, there were 1,100 terms and 660 were in the most relevant $60 \%$. After nonspecific terms were eliminated, the remaining 449 terms were used for cluster analysis (Online Supplementary Material 8; - Fig. 7).

\section{Comparison of the Clustering Groups over the Different Periods}

The first two periods seem to be similar to each other in both numbers and content of the clusters. In the third period, 


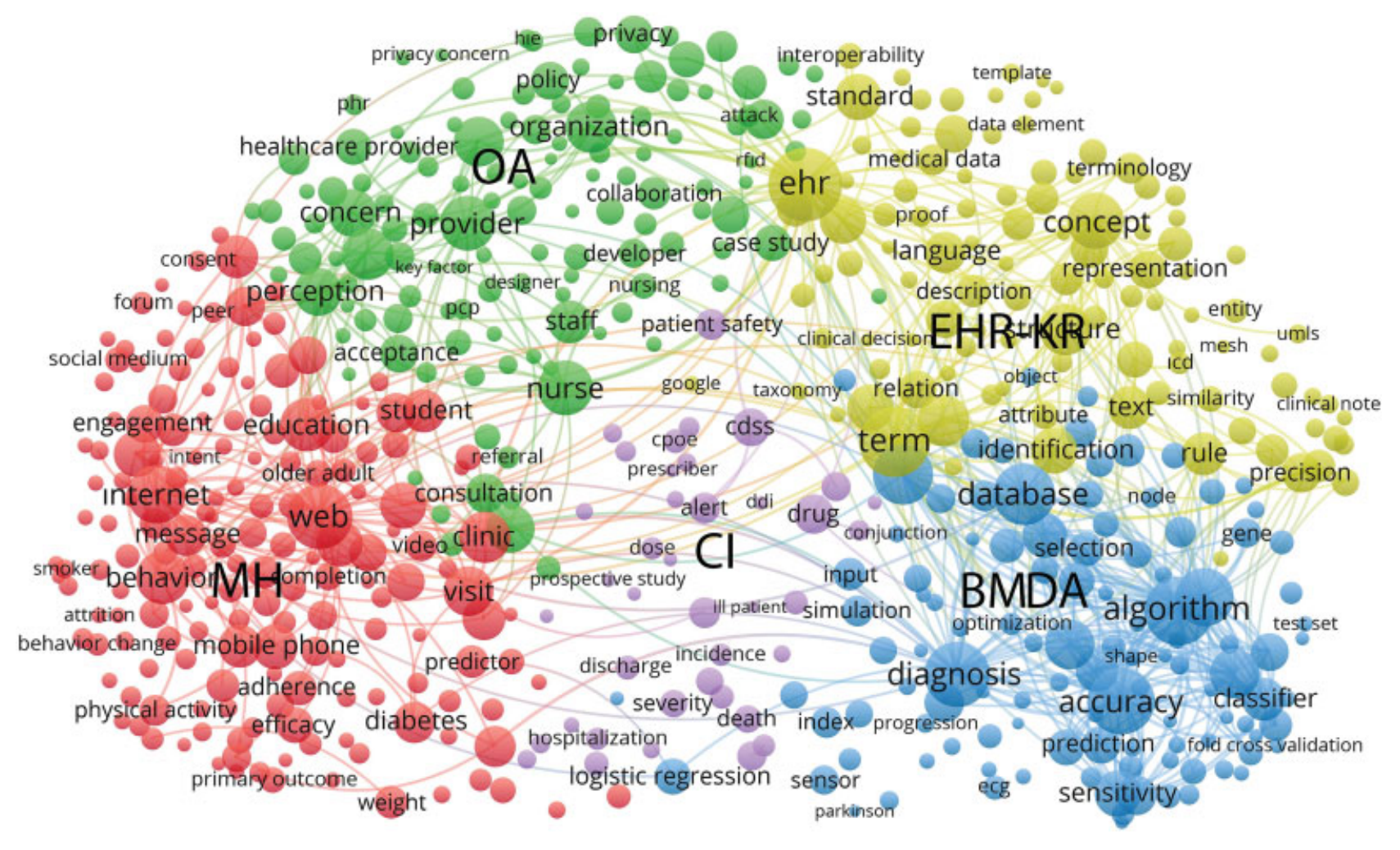

accelecometer

Fig. 3 Cluster map of the terms obtained by text mining over 12 years (2006-2017) of articles on different topics from core MI journals. Topics are colored as: red: MH, Mobile Health, green: OA, Organizational Aspects Of Health Information Systems, yellow: EHR-KR, EHR and knowledge representation, blue: BMDA, Biomedical Data Analysis, and purple: $\mathrm{Cl}$, Clinical Informatics.

some terms like "mobile phone," “mobile apps," and "message" appear in the cluster, which we named telehealth in the previous periods. We named this cluster as mobile health in periods three and four. In the third period, a relatively small clinical informatics cluster appears and persists in the fourth period. In the fourth period, we observe a rearrangement of clusters. "EHR," "integration," "standards," "information systems," "privacy," "workflow," "security," and "documentation" terms become detached from the previous "Organizational Aspects of Health Information Systems" cluster and shift to the previous "Knowledge Representation" cluster. We named this new composition as "EHR and Knowledge Representation." A general view of clusters according to the four time periods is presented in -Fig. 8 .

\section{Discussion}

According to our cluster analysis for journals, the core MI journals we identified overlap only partially with the WoS MI category. Ten journals in the WoS MI category were identified as belonging to other clusters, and two journals in the "Healthcare Sciences and Services" category were identified as belonging to the MI cluster. Another classification of scientific journals was made by Science-Metrix. ${ }^{31,32}$ Its categories were modeled on those of existing journal classifications, and their groupings of journals acted as "seeds" or attractors for journals in the new classification. Individual journals were assigned to single, mutually exclusive categories via a hybrid approach combining algorithmic methods (using citation data and author addresses) and expert judgment. ${ }^{31}$ MI category contains 30 journals. Three journals in our MI cluster were published after this classification, so they are not present in the list. Other three well-known MI journals in our MI cluster (CIN -Computers Informatics Nursing, Health Information Management Journal, and Informatics for Health and Social Care) are also not present in the list. The list contains eight medical education journals, a few MI journals which are not included by WOS, and a few journals which are in different clusters in our clustering results. Our results only partially overlap with Science-Metrix classification.

Our cluster analysis revealed 47 clusters of journals. Considering the six related clusters, according to our analysis, the "Bioinformatics" and "Statistics" clusters were close to each other, whereas, to our surprise, they did not have a close relationship with the MI cluster. However, "Health management," "Information Management," and "Biomedical Engineering: Imaging and Information Technology" clusters are three close neighbors of the MI cluster. Although they are under the "Biomedical Informatics" umbrella, the MI and Bioinformatics scientific communities have divergent features related to their scientific conferences and journals. Deeper insight into this situation as well as some suggestions to increase communication between these scientific fields have been discussed previously. ${ }^{33,34}$ On the other hand, with 


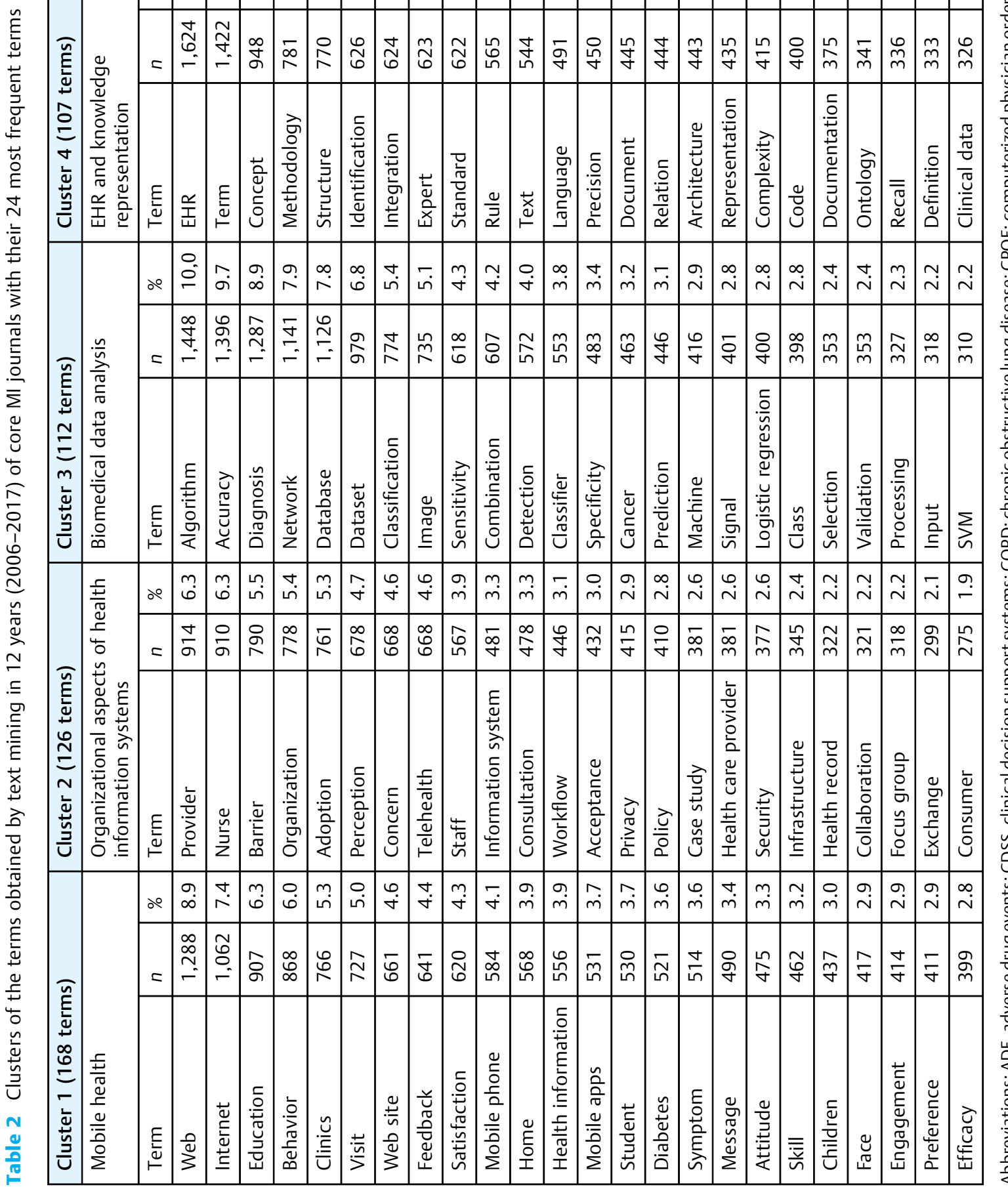

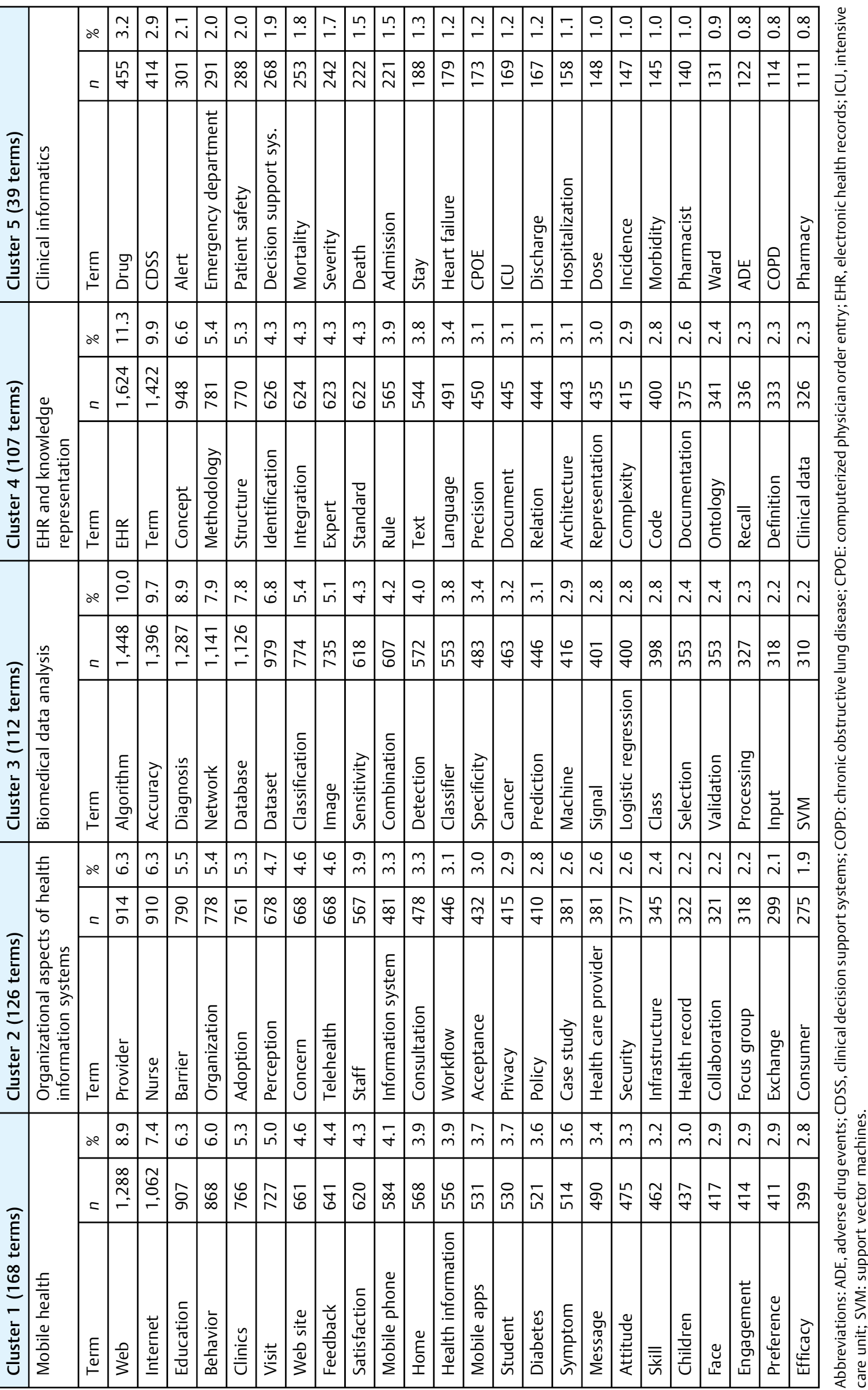




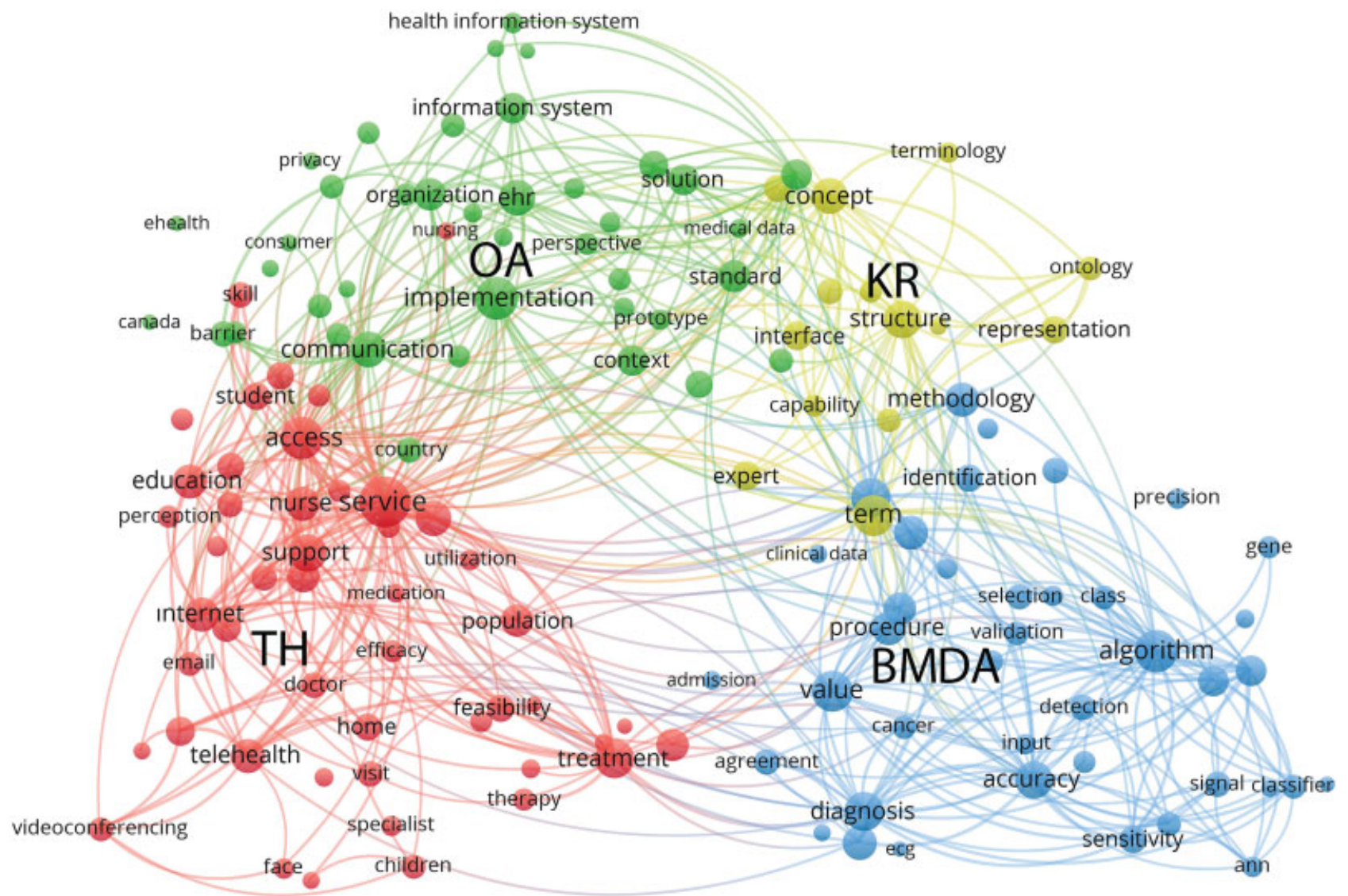

Fig. 4 Cluster map of the terms obtained by text mining from articles from core MI journals published in 2006-2008. Group topics involve: red: TH, Telehealth, green: OA, Organizational Aspects of Health Information Systems, yellow: KR, Knowledge Representation, and blue: BMDA, Biomedical Data Analysis.

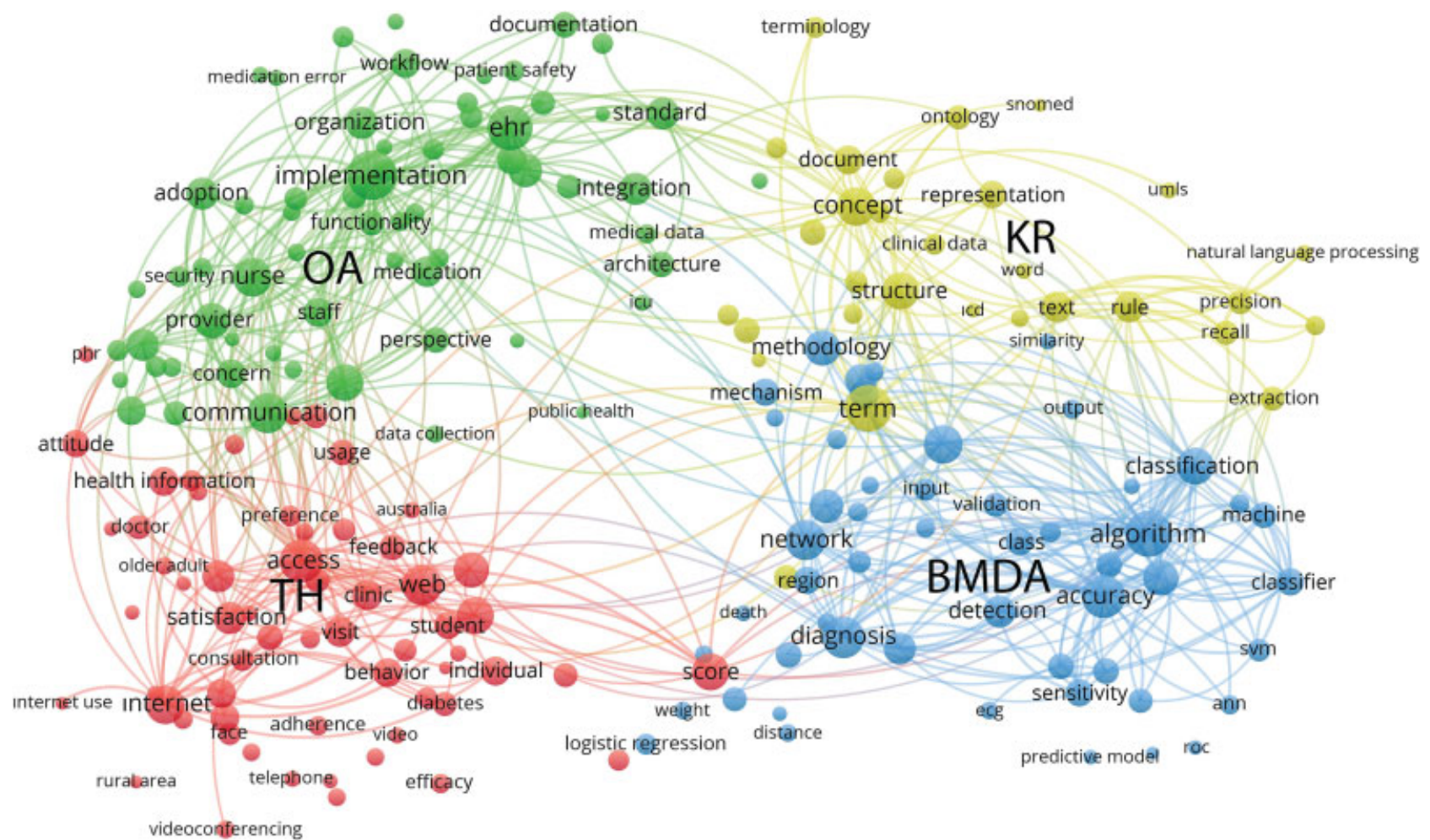

Fig. 5 Cluster map of the terms obtained by text mining from articles of core MI journals published in 2009-2011. Topics are colored as: red: TH, Telehealth, green: OA, Organizational Aspects of Health Information Systems, yellow: KR, Knowledge Representation, and blue: BMDA, Biomedical Data Analysis. 


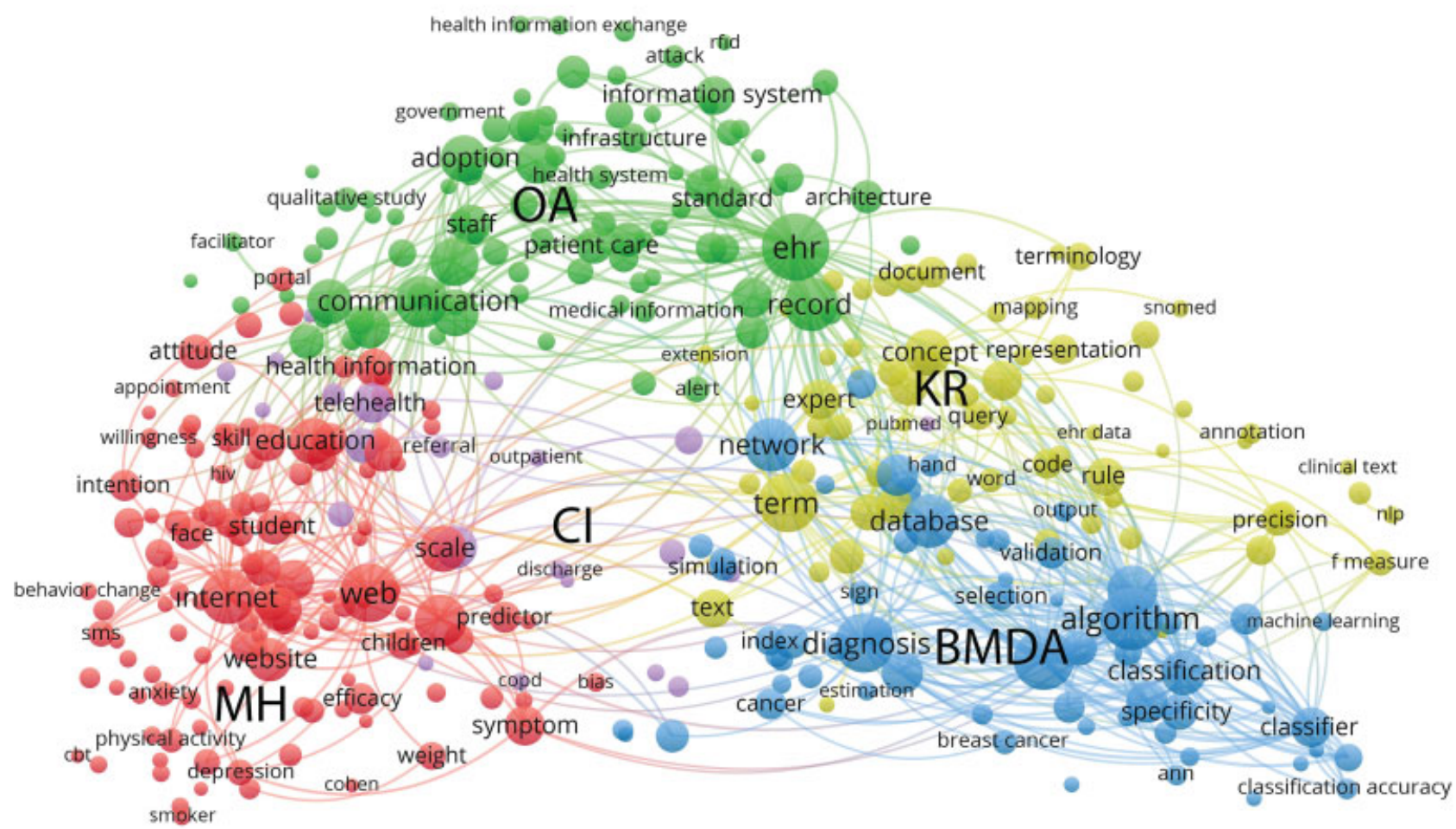

Fig. 6 Cluster map of the terms obtained by text mining from articles of core MI journals published in 2012-2014. Topics: red: MH, Mobile Health, green: OA, Organizational Aspects of Health Information Systems, yellow: KR, Knowledge Representation, blue: BMDA, Biomedical Data Analysis, and purple: $\mathrm{Cl}$, Clinical Informatics.

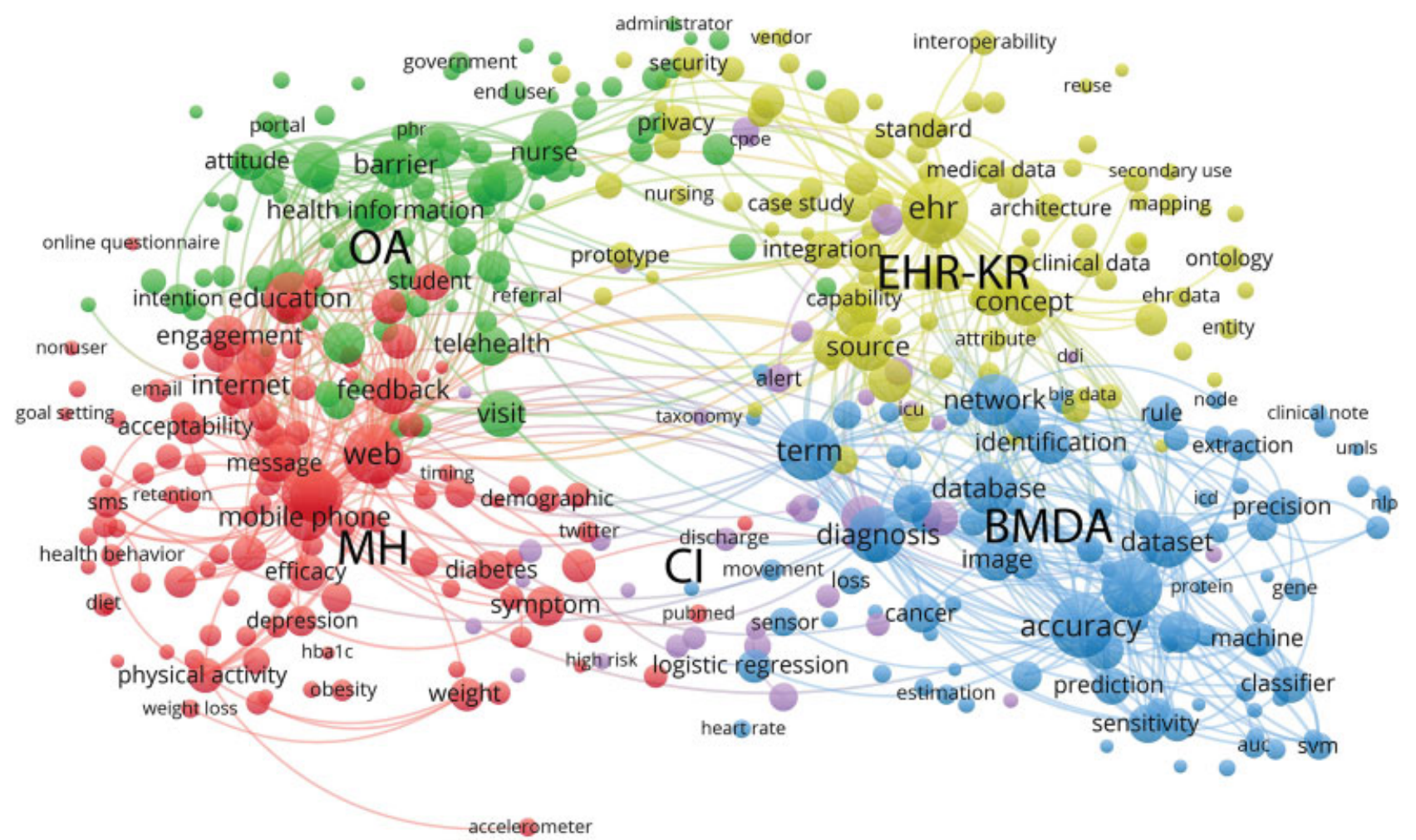

Fig. 7 Cluster map of the terms obtained from articles published in 2015-2017. Red: MH, Mobile Health, green: OA, Organizational Aspects of Health Information Systems, yellow: EHR-KR, EHR and Knowledge Representation, blue: BMDA, Biomedical Data Analysis, and purple: Cl, Clinical Informatics. 


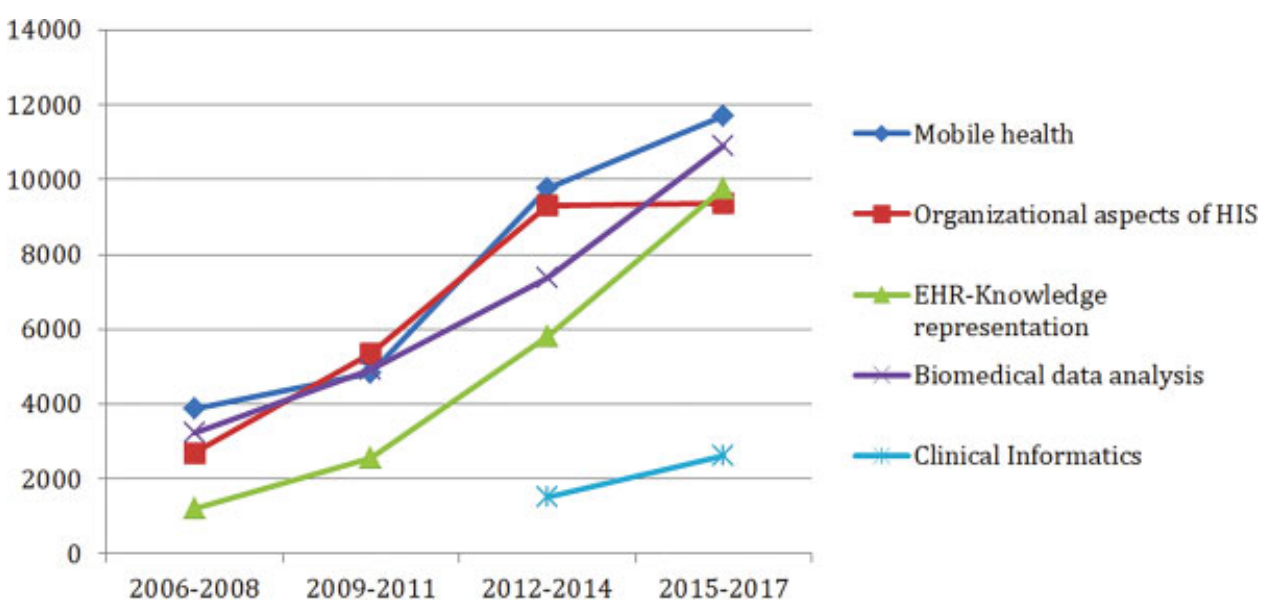

Fig. 8 Graphical representation of the number of occurrences of terms in each cluster according to time periods. The numbers show the sum of the numbers of usage of the terms (the term was used in how many articles) in each cluster. The mobile health cluster was called telehealth in the first two periods and mobile health in the third and fourth periods. EHR-Knowledge Representation cluster was called Knowledge Representation until the fourth period.

increasing efforts to integrate molecular data with those from electronic health records, we can expect a closer relation between bioinformatics and MI to develop in the future.

Although there is only some overlap between the MI cluster as identified in this analysis with the WoS MI category, in our opinion our clustering analysis results are more reasonable in some respects. For example, journals like "Statistical Methods in Medical Research" and "Statistics in Medicine" assigned to the WoS MI category are clearly not MI journals, and somehow conflate the well-known fact that statistical analyses are frequently reported in informatics papers with the notion that journals focused on statistical methods in medicine are likely to be informatics-related, which is clearly not the case. On the other hand, a journal can be assigned to more than one category in the WoS categorization system. Because our clustering method assigns each journal to only one cluster, some journals-such as "Computer Methods and Programs in Biomedicine"-with relatively low MI content may be assigned to other clusters and not to the MI cluster.

We also tried to compare our journal clustering results with previous studies, although comparisons of this type are problematic, among other reasons, because of the different time periods covered and ever-shifting professional practices, which often make the content of the journals change over time. $^{14}$

- Using MeSH: Some of studies using MeSH have not mentioned journal names and other such studies have produced very questionably relevant results. For example, according to one study, the journals considered to be the most prominent ones publishing MI articles are "Proceedings of IEEE Engineering in Medicine and Biology Society Conference," "IEEE Transactions on Image Processing," and "Medical Physics." 9 This clear overspecific focus on journals that emphasize engineering or computational methodologies for analysis and design, and not the informatics methods used in most studies (which may, how- ever, implicitly rely on engineering and computational implementations), may arise from using MeSH inappropriately for defining MI articles, and related to problems in the MeSH indexing structure and implementations. For example, in one article, $63 \%$ of the articles, indexed by the telemedicine term, were found to have not been indexed by $\mathrm{MI}$ or bioinformatics terms. ${ }^{35}$ According to another study, the sensitivity of MeSH-term-based search is 60\% and one-third of the obtained articles were found to be irrelevant to the intended subject. ${ }^{36}$ On the other hand, searching by MeSH terms has the capability of detecting some important papers in other journals because of core and scatter phenomenon. Core and scatter is the distinctive pattern of concentration and dispersion that appears in collections of papers when relative frequencies of entities are counted. In the context of mapping specialties, core and scatter has a significant effect on gathering a collection of papers to cover the specialty. On the one hand, it is usually easy to find a group of highly relevant papers that cover the core of the specialty, but on the other, it becomes increasingly laborious to gather all papers with some significant relevance, and impossible to gather all papers that are marginally relevant to the specialty. ${ }^{15}$ According to a MeSH-term-based research, 30 journals represented the first third of the total published articles in the MI field. ${ }^{11}$

- Composing a core MI journal set by expert opinion: In another study published in 2017, the authors defined which journals "belonged" to the MI category according to expert opinions. ${ }^{12}$ They made a list of $36 \mathrm{MI}$ journals. This list includes all of our core MI journals except for two very new Journal of Medical Internet Research (JMIR) journals. According to their classification, "Computer Methods and Programs in Biomedicine" and "IEEE Journal of Biomedical and Health Informatics" are also in the group of MI journals. The remaining journals were MI journals or proceedings which are not covered by ISI, or health information management journals which are not 
covered by ISI or in the health management cluster in our classification.

- Clustering journals by co-citation data to determine a core MI journal set: The study, which was based on the cocitation method, is rather old and therefore hardly comparable to our study (1993-1995 vs. 2013-2017). ${ }^{13}$

- Text mining of abstracts and clustering journals by the help of the obtained terms to determine a core MI journal set: In a study published in 2009, the results were similar except they included "Computer Methods and Programs in Biomedicine," and the IEEE Journal of Biomedical and Health Informatics (under its previous name "IEEE Transactions on Information Technology in Biomedicine") in the set of core MI journals, and they did not include two telemedicine journals. Naturally, new journals such as "Applied Clinical Informatics" were not included in this study. The difference may be due to a difference in the way in which research methods for clustering were applied-such as clustering based on terms versus clustering based on citations or by different time periods of the studies (1993-2008 vs. 2013-2017). ${ }^{14}$

It seems that clustering by text mining gives the closest results to our method. The main difference of the two methods is that text mining presents a classification based on the use of words, terms, and concepts, whereas bibliographic coupling presents a classification based on the flow of scientific information, knowledge, and ideas. In other words, the first method answers the question of "how can one classify journals according to the use of terminology," whereas the latter method answers the question of "how can one classify journals according to similarity of information or knowledge, which they present." The latter, of course, depends crucially on the definition of "similarity" and how it is computed in relation to the individual and groups of items being classified-journals in this case. So, the preferred method may change according to the point of view, choice of methods, and even the techniques of implementation chosen by a researcher.

We preferred using text mining instead of using a controlled vocabulary or author keywords for analyzing MI subjects. The advantage of text mining is its objectivity (absence of intervention by an author or an indexer) and capability of detecting new terms. On the other hand, it has a disadvantage of resulting in a disorganized bunch of terms. In a controlled vocabulary, such as MeSH, synonyms are collected under the same term and the terms are organized ontologically. Results of text mining require some error prone manual work to collect synonyms under the same umbrella and interpretation of the results is more difficult.

As a result of the terms obtained by text mining, the five clusters of terms in the examined period 2006 to 2017 can be described as follows.

- "Mobile Health": Typical terms in this cluster are "web," "education," “mobile phone," “home," "mobile apps," "diabetes," and "message." This cluster is the result of the effects of introducing new mobile technologies in health care applications. It seems that there is substantial research on mobile apps, homecare, online education, and diabetes. We were observing this trend, but it is still surprising to see them as a separate big cluster. This cluster was called "Telehealth" in the first two periods (2006-2011). By the appearance of the "mobile health" term, the "telehealth" term migrated to the "Clinical Informatics" cluster in the third period (2012-2014), and finally to the "Organizational Aspects" cluster in the fourth period (2015-2017). Probably this change corresponds to the increasing integration of telehealth practices in routinely used information systems.

- "Organizational Aspects of Health Information Systems": we observed terms such as "provider," "nurse," "barrier," "organization," “adoption,” "perception,” “concern,” “telehealth," and "privacy" in this cluster. This cluster represents an important aspect of MI. It reflects the studies on the relation of information systems with organizations and people. This cluster is the only one which ceased to enlarge in the last period (2015-2017). This may be a result of the widespread use of health information systems in health care institutions and the increasing acceptance of them, which means that early introduction problems are no longer central, while often-heard complaints about the inadequacies of health care systems and their detriments to clinical practice and workflows are not included under organizational aspects, possibly due to the socio-economic complexities involved and the sensitivities of industry and governments to such complaints.

- "EHR and Knowledge Representation": Terms such as "EHR," "term," “concept," “methodology," "structure," “identification," and "integration" were most prominent in this cluster. This cluster also reflects an important field of MI. The "EHR" term was in the "Organizational Aspects" cluster at the beginning, but it migrated to this cluster in the last period (2015-2017). It may show that the acceptance phase of EHR as a concept is coming to an end, and researchers are concentrating on the technical aspects of EHR.

- "Biomedical Data Analysis": Typical terms in this cluster are "algorithm," “diagnosis," "dataset," “classification," “image," “detection," “classifier," "prediction," and "machine." It seems that this cluster contains mostly terms related to theoretical as well as a few practical aspects of decision support and data analysis systems for biomedical research, including machine learning and imaging informatics.

- “Clinical Informatics": We observe "drug," “CDSS," “alert," "emergency department," "patient safety," “admission," "heart failure," and "CPOE" as typical terms in this cluster. This is a new and relatively small cluster, and reflects the ultimate aim of MI, i.e., to support better health care services, though one might expect some of the organizational issues that are arising in acceptance of such systems to migrate to this cluster in the future.

Studying MI terms in the literature has been performed in several studies. However, we found only two studies, which also dealt explicitly with the clustering of these terms. In the 
first study, MeSH terms for articles published in $20 \mathrm{MI}$ journals in the period of 1995 to 1999 were clustered. The authors found eight clusters, namely "Imaging Techniques," "Diagnostic Imaging," "Science and the Art of Medicine," "Statistical Analysis," Biochemical Communications," "Cognitive and Physiological Communication Concepts," "Immunology," and "Molecular Genetics." Results of this article are not comparable to those from our study because of differences in research methods (MeSH terms vs. text mining) and the many technological and practice changes between the periods studied (1995-1999 vs. 2006-2017). ${ }^{16}$

In another study, abstracts of $16 \mathrm{MI}$ journals published in the period of 1993 to 2008 were text mined and the obtained terms were clustered. The authors obtained three main clusters. They did not name them but described: "Cluster 1 appears to deal mainly with health information systems, their application, evaluation, and organization. An investigation of cluster 1.3 showed that this cluster contains many documents describing user evaluations of health information systems. Cluster 2 deals mainly with medical knowledge representation in the form of clinical guidelines, ontologies, and databases. Also included is a subcluster dealing more specifically with the analysis of medical language. Cluster 3 deals with data analysis, with subclusters for classification techniques and statistical modeling, signal analysis, microarray analysis, and the field of image analysis." 14 These clusters are similar to our clusters "Organizational Aspects of Health Information Systems," "EHR and Knowledge Representation," and "Biomedical Data Analysis," respectively. We found two additional clusters in our analysisnamely "Mobile Health" and "Clinical Informatics." When we consider that these two clusters were not present in articles from our first two periods, we can conclude that these subjects represent rising subfield trends which are likely to continue.

Our study has several limitations, mostly due to the complex nature of our research subject.

One limitation is that we have limited ourselves by only considering sources, which are indexed by WoS. Therefore, our clustering approach did not include proceedings of important MI meetings such as MEDINFO, MIE, and the AMIA Annual Symposium. There are also a few MI journals which are not covered by ISI, and we could not include them, because our clustering is based on reference data in the ISI database. In addition, we are aware of the fact that MI articles are also published in a wide range of journals, often in related but frequently only loosely related disciplines in their relationship to MI. However, since we are convinced that most of high-quality MI research is communicated through core MI journals, we nevertheless wanted to focus on these journals.

The clustering method itself imposes several limitations besides the foundational one of choosing a similarity measure for the clustering. The size of the clusters can be chosen to be either smaller or larger, and to include fewer or more journals. This depends on just how "loosely and generally" one wishes to define such a heterogeneous and complex field of study and application as MI. There has been long-standing discussion in the discipline initiated by van Bemmel ${ }^{37}$ and others ${ }^{38-40}$ on the very definition of MI as art versus science and implicitly the problems of clinical practice versus bio- medical inquiry, as well as the technology and engineering of systems that bridge the two. Bearing this in mind, a short empirical study like the present one can barely scratch the surface of some of the deeper issues that arise in trying to clarify how clustering publications in the literature is used to help "ground" conceptualizations of our field in the bibliographic evidence that is constantly accumulating. This is why, among other considerations, the size of the MI journal cluster in our present study was adjusted according to our personal opinions, with this decision obviously having a subjective component, as do most of the empirical choices made in applying clustering methods, which has, after all, a high component of subjective "guessing." 41

We examined only five years of data for clustering the journals. It may also be considered as a limitation of the study. The WoS only permits downloads of data to a maximum of 100,000 articles per search. Because the 2012 search resulted in a larger number of articles, we limited our analysis to the years: 2013 to 2017 .

The text mining method still depends on important choices of parameters that are largely subjective, the attribution of labels to groups is also a matter of expert opinion, and needs substantial human intervention. In spite of selecting $60 \%$ of the most relevant terms, we observed a lot of terms which do not give us clues about research subjects and had to manually exclude them from the analysis. So, this termelimination process was done according to experiences and perceptions or opinions of the authors, introducing hard-toassess subjectivity, though it does represent state-of-the-art methods. The term-elimination process is largely reproducible, because the word lists are given as supplementary material. However, if new terms arise in future, these lists may not be helpful for them.

We would like to also state that detected changes in number and content of the clusters by time can be affected by various factors. First, the resolution values for each clustering were selected empirically. This can affect the number and content of clusters. Second, changes in the use of the terms are possible in scientific writing. A concept may be named differently a few years later.

\section{Conflict of Interest}

Both of the authors are or have been in editorial boards of various journals. Most of these journals are in the field of MI.

\section{Acknowledgment}

The authors would like to thank Casimir Kulikowski for his support during the initial stage of manuscript preparation. Through his edits, he not only turned the text into a much better readable one, but also helped to further reflect on methodological approach and on its limitations.

\section{References}

1 Musen MA, van Bemmel JH. Challenges for medical informatics as an academic discipline: workshop report. Yearb Med Inform 2002;(01):194-197 
2 Hersh W. A stimulus to define informatics and health information technology. BMC Med Inform Decis Mak 2009;9:24

3 Haux R. On determining factors for good research in biomedical and health informatics. Some lessons learned. Yearb Med Inform 2014;9:255-264

4 Mantas J, Ammenwerth E, Demiris G, et al; IMIA Recommendations on Education Task Force. Recommendations of the International Medical Informatics Association (IMIA) on education in biomedical and health informatics. First revision. Methods Inf Med 2010;49(02):105-120

5 Chen ES, Sarkar IN. *informatics: identifying and tracking informatics sub-discipline terms in the literature. Methods Inf Med 2015;54(06):530-539

6 Bernstam EV, Smith JW, Johnson TR. What is biomedical informatics? J Biomed Inform 2010;43(01):104-110

7 Sittig DF. Identifying a core set of medical informatics serials: an analysis using the MEDLINE database. Bull Med Libr Assoc 1996; 84(02):200-204

8 Lavallie DL, Wolf FM. Publication trends and impact factors in the medical informatics literature. AMIA Annu Symp Proc 2005:1018

9 Deshazo JP, Lavallie DL, Wolf FM. Publication trends in the medical informatics literature: 20 years of "Medical Informatics" in MeSH. BMC Med Inform Decis Mak 2009;9:7

10 Elkin PL, Brown SH, Wright G. Biomedical informatics: we are what we publish. Methods Inf Med 2013;52(06):538-546

11 Lyu PH, Yao Q Mao J, Zhang SJ. Emerging medical informatics research trends detection based on MeSH terms. Inform Health Soc Care 2015;40(03):210-228

12 Wang L, Topaz M, Plasek JM, Zhou L. Content and trends in medical informatics publications over the past two decades. Stud Health Technol Inform 2017;245:968-972

13 Morris TA, McCain KW. The structure of medical informatics journal literature. J Am Med Inform Assoc 1998;5(05):448-466

14 Schuemie MJ, TalmonJL, Moorman PW, Kors JA. Mapping the domain of medical informatics. Methods Inf Med 2009;48(01):76-83

15 Morris SA, Van der Veer Martens B. Mapping research specialties. Annu Rev Inform Sci Tech 2008;42(01):213-295

16 Kessler MM. Bibliographic coupling between scientific papers. Am Doc 1963;14(01):10-25

17 Marshakova-Shaikevich I. System of document connections based on references. Nauchno Tekhnicheskaya Informatsiya Seriya 2Informatsionnye Protsessy i Sistemy 1973;6:3-8

18 Small H. Co-citation in the scientific literature: a new measure of the relationship between two documents. J Am Soc Inf Sci 1973; 24(04):265-269

19 Boyack KW, Klavans R. Co-citation analysis, bibliographic coupling, and direct citation: which citation approach represents the research front most accurately? J Am Soc Inform Sci Tech Arch 2010;61(12):2389-2404

20 Klavans R, Boyack KW. Which type of citation analysis generates the most accurate taxonomy of scientific and technical knowledge? J Assoc Inf Sci Technol 2017;68(04):984-998

21 Morris TA. Structural relationships within medical informatics. Proc AMIA Symp 2000:590-594

22 González LM, García-Massó X, Pardo-Ibañez A, Peset F, DevísDevís J. An author keyword analysis for mapping sport sciences. PLoS One 2018;13(08):e0201435
23 VOSviewer version 1.6.8. Available at: http://www.vosviewer. com. Accessed August 20, 2018

24 Waltman L, van Eck NJ. A smart local moving algorithm for largescale modularity-based community detection. Eur Phys J B 2013; 86(11):471

25 Perianes-Rodriguez A, Waltman L, Van Eck NJ. Constructing bibliometric networks: A comparison between full and fractional counting. J Informetrics 2016;10(04):1178-1195

26 Van Eck NJ, Waltman L. How to normalize co-occurrence data? An analysis of some well-known similarity measures. J Am Soc Inf Sci Technol 2009;60(08):1635-1651

27 Van Eck NJ, Waltman L. Visualizing bibliometric networks. In: Ding Y, Rousseau R, Wolfram D, eds. Measuring Scholarly Impact: Methods and Practice. Berlin: Springer; 2014:285-320

28 Van Eck NJ, Waltman L. VOSviewer Manual. Available at: http:// www.vosviewer.com/download/f-z2X2.pdf. Accessed August 20, 2018

29 Apache OpenNLP library. Available at: http://opennlp.apache.org. Accessed August 20, 2018

30 Van Eck NJ, Waltman L. Text mining and visualization using VOSviewer. ISSI Newsletter 2011;7(03):50-54

31 Archambault É, Beauchesne $\mathrm{OH}$, Caruso J. Towards a multilingual, comprehensive and open scientific journal ontology. In: Noyons B, Ngulube P, Leta J, eds. Proceedings of the 13th International Conference of the International Society for Scientometrics and Informetrics (ISSI), Durban, South Africa; 2011:66-77

32 Science-Metrix Classification of Scientific Journals. Sixth public release: 2006-03-31 (v1.06). Available at: http://science-metrix. com/sites/default/files/science-metrix/sm_journal_classification _106_1.xls. Accessed October 19, 2018

33 Maojo V, García-Remesal M, Bielza C, Crespo J, Perez-Rey D, Kulikowski C. Biomedical informatics publications: a global perspective: part I: conferences. Methods Inf Med 2012;51(01):82-90

34 Maojo V, Garcia-Remesal M, Bielza C, Crespo J, Perez-Rey D, Kulikowski C. Biomedical informatics publications: a global perspective. Part II: journals. Methods Inf Med 2012;51(02):131-137

35 Geissbuhler A, Hammond WE, Hasman A, et al. Discussion of "Biomedical informatics: we are what we publish". Methods Inf Med 2013;52(06):547-562

36 Saka O, Gülkesen KH, Gülden B, Koçgil OD. Evaluation of two search methods in PubMed; the regular search and search by MeSH terms. Acta Inform Med 2005;13(04):180-183

37 van Bemmel JH. Medical informatics, art or science? Methods Inf Med 1996;35(03):157-172, discussion 173-201

38 Martin-Sanchez FJ, Lopez-Campos GH. The new role of biomedical informatics in the age of digital medicine. Methods Inf Med 2016; 55(05):392-402

39 Al-Shorbaji N, Bellazzi R, Gonzalez Bernaldo de Quiros F, et al. Discussion of "The New Role of Biomedical Informatics in the Age of Digital Medicine". Methods Inf Med 2016;55(05):403-421

40 Haux R, Kulikowski CA, Bakken S, et al. Research strategies for biomedical and health informatics. Some thought-provoking and critical proposals to encourage scientific debate on the nature of good research in medical informatics. Methods Inf Med 2017;56 (Open):e1-e10

41 Watanabe S. Knowing and Guessing: a Quantitative Study of Inference and Information. New York, NY: John Wiley \& Sons Inc; 1969 